\title{
Uma análise do rendimento escolar por área de conhecimento como forma de desmistificação de estereótipos de gênero em Computação
}

\author{
Maria Alice N. Silva $^{1}$, Thalia S. Santana ${ }^{1}$, Adriano H. Braga ${ }^{1}$ \\ ${ }^{1}$ Instituto Federal Goiano - Campus Ceres (IF GOIANO) \\ Caixa Postal 51 - 76.300-000 - Ceres - GO - Brasil \\ maria.nunes@estudante.ifgoiano.edu.br, \\ \{adriano.braga, thalia.santana\}@ifgoiano.edu.br
}

\begin{abstract}
This work analyzes the school performance by gender of students in the technical course in Computer Science into six areas of knowledge. A database was developed, with information from 255 students from all grades of the technical course in Computers for the Internet, integrated with high school of a Federal Institute in 2019, 161 boys and 94 girls. The analysis of the average grades of six knowledge areas grouped by gender showed that female students have a slightly higher average than male students in all areas of knowledge.
\end{abstract}

Resumo. O presente estudo tem como objetivo analisar o rendimento escolar por gênero de estudantes do curso técnico em Informática para Internet em seis áreas de conhecimento. Foi elaborado um banco de dados, com informações de 255 estudantes de todas as séries do curso técnico em Informática para Internet integrado ao ensino médio do ano de 2019 de um Instituto Federal, sendo 161 meninos e 94 meninas. A análise das médias de notas de seis áreas de conhecimento agrupadas por gênero demonstrou que as estudantes do gênero feminino possuem média ligeiramente superior ao gênero masculino em todas as áreas de conhecimento.

\section{Introdução}

A educação superior no Brasil é cursada majoritariamente por mulheres, sendo cerca de 57,2\% de acordo com dados do Censo da Educação Superior de 2016 (INEP, 2017). Contudo, esses números tendem a cair drasticamente quando analisa-se cursos da área de informática, como por exemplo no curso de Ciência da Computação da Universidade de São Paulo (USP) que, no mesmo ano verificou que apenas $15 \%$ de ingressantes são do gênero feminino (SANTOS, 2017). Tais índices compõem um cenário de desequilíbrio de gênero em profissões relacionadas às ciências exatas, o qual perpassa por todo o ocidente (LOUZADA et al., 2019).

Estatísticas como essa são resultantes de reflexos anteriores, a exemplo do Programa Internacional de Avaliação de Alunos - PISA (PISA EM FOCO, 2015) em que menos de $5 \%$ das meninas de 15 anos de idade que possuem expectativas profissionais pensam em seguir por áreas de engenharia e computação. Um dos 
apontamentos do estudo relata que existe uma menor autoconfiança entre as meninas, principalmente no que tange à suas habilidades de resolução de problemas, como de ciências ou matemática, e quando estimuladas a pensar cientificamente, possuem desempenho inferior ao do público masculino. Contudo, quando meninos e meninas são igualmente confiantes, não há diferença de gênero em desempenho.

Com o intuito de amenizar o impacto destes estereótipos com as estudantes do gênero feminino na área de Informática, o projeto Meninas Digitais no Cerrado foi criado no ano de 2016, para transformar o Campus Ceres do IF Goiano em uma instituição parceira da Sociedade Brasileira de Computação (SBC) por meio de seu programa nacional homônimo (Meninas Digitais), existente desde 2011. Sendo um campus de origem agrária no interior do Estado de Goiás, dentre as atividades desenvolvidas busca-se valorizar perfis femininos de êxito na computação, muitas vezes com sua participação histórica omitida, a fim de prover visibilidade e demonstrar a real importância de figuras pioneiras da área, estimulando às participantes no que tange a identificação pessoal com carreiras tecnológicas, além de também tecer estudos que possam colaborar no entendimento de fatores que as afastam (LOUZADA et al., 2019).

O objetivo do presente trabalho é analisar o rendimento escolar por gênero, de 255 estudantes de todas as turmas do Curso Técnico em Informática para Internet do IF Goiano - Campus Ceres do ano de 2019, em seis áreas de conhecimento. Com este estudo pretende-se responder a seguinte questão de pesquisa: "Existe diferença no rendimento acadêmico entre os gêneros masculino e feminino por área de conhecimento dentro de um curso de nível técnico em informática?’. O presente artigo contribui para a análise e produção de dados que validem o desenvolvimento de ações de motivação, capacitação e empoderamento de meninas do ensino médio técnico, visando maior ingresso feminino no ensino superior na área de Ciência e Tecnologia, a exemplo do projeto Meninas Digitais no Cerrado, existente na instituição de origem dos autores.

Este artigo está organizado da seguinte maneira: a seção 2 apresenta os trabalhos relacionados; na seção 3 encontram-se os materiais e métodos empregados neste estudo; a seção 4 contém os resultados e discussão; na seção 5 estão as considerações finais; na seção 6 constam os agradecimentos; e por último, as referências bibliográficas.

\section{Trabalhos Relacionados}

Esta seção descreve estudos correlatos e pesquisas ligadas ao gênero e desempenho escolar em cursos da área de informática. Resende, Gomes e Berardi (2020) analisaram a influência da formação de grupos com diversidade de gênero e o desempenho acadêmico na disciplina de banco de dados dos cursos de Engenharia da Computação e Sistemas de Informação. Foi verificado que equipes com participação feminina possuíam maiores notas, ainda que o desempenho individual feminino seja inferior quando comparado ao masculino. Tal fenômeno pode estar ligado aos estereótipos de gênero que perpassam toda a trajetória feminina na computação. 
Pereira et al. (2020) verificaram o rendimento acadêmico de estudantes dos gêneros masculino e feminino do curso de ciência da computação do Instituto Federal Sudeste de Minas Gerais. O estudo demonstrou que a média do rendimento acadêmico do gênero feminino é superior ao do gênero masculino, sendo respectivamente $65,3 \% \mathrm{e}$ $57,2 \%$. Além disso, elas também possuem maior percentual de aprovação em boa parte das disciplinas do departamento de matemática, física e estatística e do departamento de ciência da computação ao cursar a matéria apenas uma vez.

Dados como os descritos na pesquisa supracitada comprovam a inexistência da diferença de desempenho entre os gêneros, mesmo em áreas tidas como "masculinas", provando que homens e mulheres são igualmente capazes. Contudo, as mulheres representam apenas 14,25\% dos ingressantes no curso analisado no período de 2007 até o primeiro semestre de 2020, esclarecendo que muito embora não exista diferença de desempenho entre os gêneros, os estereótipos acerca da computação ainda influenciam a escolha feminina de uma graduação na área.

Marques et al. (2021) trazem uma análise do desempenho acadêmico por gênero de estudantes do Curso Técnico em Informática do Instituto Federal de São Paulo entre os anos de 2016 a 2020. Conforme os dados apresentados, as estudantes do gênero feminino possuem média levemente superior aos estudantes do gênero masculino em todos os anos analisados. Ao verificar a média geral dos estudantes nos três anos do Ensino Médio também é possível observar o mesmo fenômeno.

Para entender as causas relacionadas, Teixeira e Posser (2016) contribuem no entendimento do cenário do ensino técnico em informática referente à verticalização do ensino. Os autores realizaram uma pesquisa com mais de 400 respondentes mulheres, que informaram que dentre os motivos levantados pela escolha profissional em computação, a realização de um curso técnico na área foi um dos três motivos mais citados, o que ressalta a importância de uma formação anterior para maior sentimento de pertencimento e autoconfiança no ingresso em uma graduação em Tecnologia da Informação (TI). O trabalho também aponta como razão para o número inferior de mulheres neste campo, o preconceito e estereótipo de que esta é uma área para homens.

Ainda conforme dados do Relatório do Programa Meninas Digitais 2018-2019, verifica-se que entre parte dos projetos parceiros, meninas do ensino médio participantes das ações desenvolvidas no ano de 2018 optaram por seguir carreira na área, a exemplo de ingresso no ensino superior em Computação e áreas correlatas (RIBEIRO; MACIEL; BIM, 2019). Isso demonstra a importância de atividades de incentivo de meninas em informática, a fim de minimizar a evasão estudantil feminina, motivando-as a permanecer no âmbito profissional por meio da escolha de uma graduação em TI.

Levando em consideração o exposto acima, percebe-se que o processo de escolha de cursos na área de computação por mulheres vai muito além do simples interesse pessoal, tendo em vista os estereótipos de gênero a serem enfrentados, a baixa representatividade na área, entre outras dificuldades. Todos esses fatores colaboram para a baixa presença feminina em cursos de graduação em computação. Dessa forma, o trabalho de Ribeiro e Maciel (2020) com 78 estudantes do gênero feminino do Ensino Médio Técnico em Informática do estado do Mato Grosso aponta que apenas 27\% delas 
consideram seguir carreira em TI e que o processo de escolha está fortemente pautado em razões pessoais. O estudo conclui que experiências prévias na área, tanto positivas quanto negativas, realizam forte influência na decisão de permanecer na área de computação.

Por fim, tomando como base a literatura científica acerca de gênero e rendimento acadêmico, dados do relatório de projetos parceiros do Programa Meninas Digitais 2020-2021 trazem 48,9\% das pesquisas realizadas pelos projetos parceiros têm como tema análise de dados educacionais sobre mulheres e computação (MENINAS DIGITAIS, 2021). Porém, ainda assim é possível notar uma carência de estudos que abordem a influência do rendimento escolar em cursos técnicos sob a perspectiva de gênero relacionados à uma possível escolha de cursos de graduação. Evidenciando a necessidade da discussão sobre as relações de gênero predominantes na área de informática, neste caso envolvendo estudantes do IF Goiano - Campus Ceres. Considerando aspectos de verticalização do ensino em meninas regularmente matriculadas em cursos informática, é preciso que estas sintam-se confortáveis e aptas desde o ensino técnico, o que pode possuir reflexos em seu rendimento escolar, a fim de averiguar vínculos com o prosseguimento dos estudos na graduação e consequente desenvolvimento profissional.

\section{Materiais e Métodos}

A presente pesquisa possui caráter exploratório com abordagem quantitativa acerca das notas de estudantes do Curso Técnico em Informática para Internet do IF Goiano Campus Ceres do ano de 2019. Os dados foram solicitados no departamento de registro escolar da instituição que encaminhou um arquivo contendo dados de todos os 255 estudantes de todas as turmas do Curso Técnico em Informática para Internet do ano letivo de 2019, tendo em vista que até o momento da aquisição dos dados o ano letivo de 2020 ainda não havia sido finalizado em consequência da pandemia de COVID-19.

Com intuito de observar o rendimento escolar por gênero em seis áreas de conhecimento, foi iniciada a construção do diagrama lógico para a construção de um banco de dados, em seguida, foi elaborado o Diagrama Entidade Relacionamento (DER) e por fim foi construída a base de dados física. Esta etapa foi desenvolvida visando a continuação da pesquisa e de projetos futuros, pois uma base de dados normalizada e estruturada auxilia na extração e análise dos dados.

Após a obtenção dos dados e criação da base de dados, foram feitos os processos de manipulação dos mesmos, sendo: limpeza, normalização e inserção dos dados. A limpeza é a fase em que são retirados dados que não serão armazenados no banco de acordo com a modelagem feita, a exemplo de estudantes que não possuíssem nenhuma nota. Na normalização, os dados que estavam agrupados em apenas duas tabelas, uma com os diários e outra com informações dos estudantes, foram reorganizados de acordo com o banco de dados desenvolvido. E por último, a etapa de inserção corresponde a execução de comandos insert. Todos os processos de manipulação dos dados prévios a inserção no banco de dados, foram realizados de forma manual com a ferramenta 
Planilhas Google, já para a inserção dos dados no banco foi utilizada a linguagem Structured Query Language (SQL) e o SGBD MySQL Workbench.

Com a base de dados pronta e populada foram calculadas as médias e o desvio padrão de cada gênero nas seis áreas de conhecimento, conforme mostra a Figura 1. Ciências da Natureza (1) abrange as disciplinas de biologia, física e química dos três anos do ensino médio. Matemática (2) corresponde a disciplina de matemática de todos os anos do ensino médio. Ciências Humanas (3) representa as disciplinas de filosofia, geografia, história e sociologia de todos os anos do ensino médio. Linguagens e Códigos (4) corresponde às matérias de artes, língua portuguesa, inglês e espanhol de todos os anos do ensino médio. Núcleo Articulador (5) compreende as disciplinas de fundamentos de informática e operação de computadores, metodologia científica, e administração e empreendedorismo. Por fim, Núcleo Profissional de Informática (6) são as disciplinas de lógica de programação, banco de dados, manutenção de computadores, desenvolvimento web, redes de computadores, técnicas avançadas de desenvolvimento web, e desenvolvimento de projetos e sistemas.

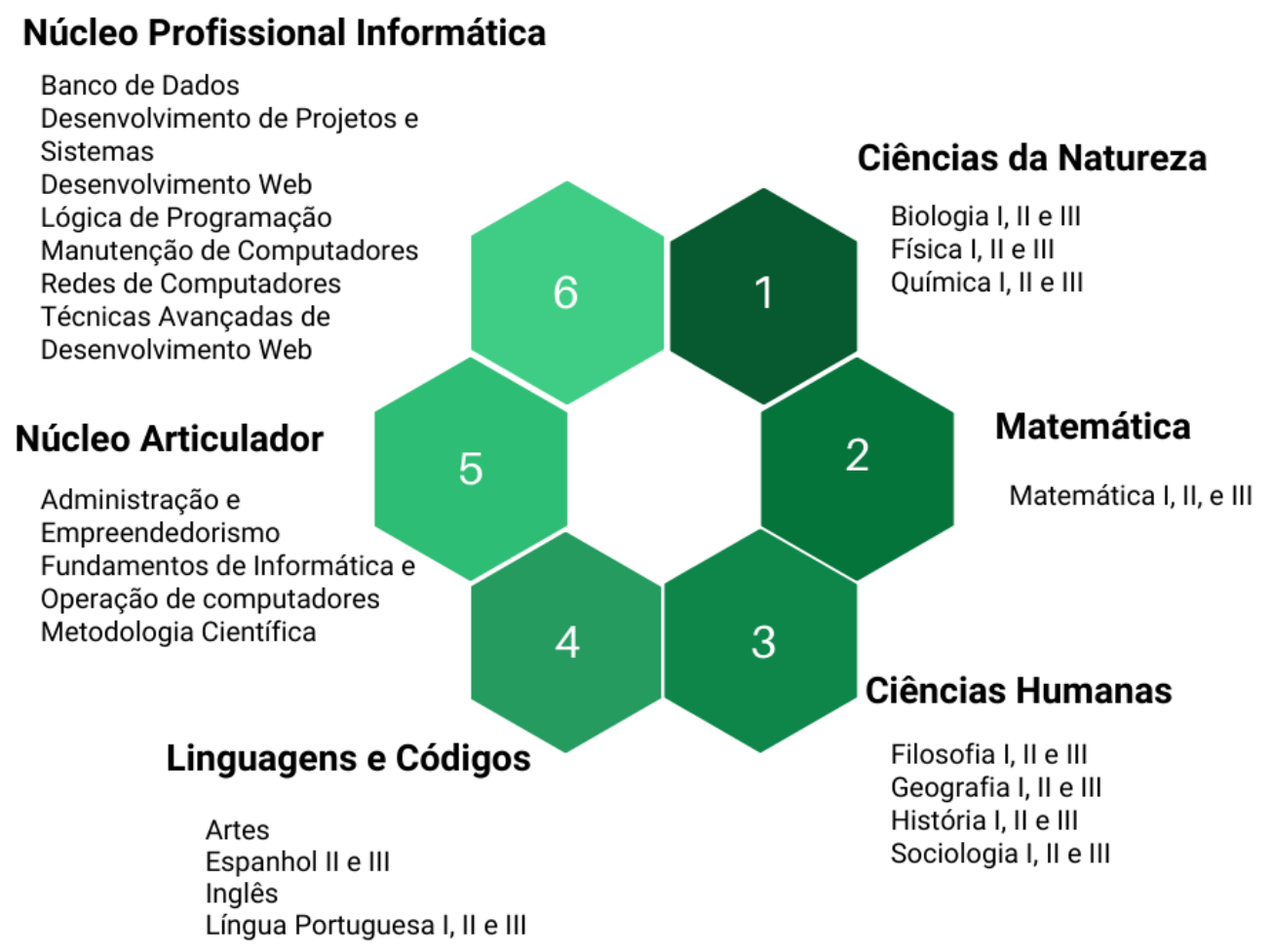

Figura 1. Diagrama das disciplinas de cada área de conhecimento. Fonte: Própria.

\section{Resultados e Discussão}

Do total de 255 estudantes analisados, 161 tratavam-se de discentes do gênero masculino, $(63,14 \%)$ e 94 do gênero feminino (36,86\%), os quais estavam divididos nas turmas de $1^{\mathrm{a}}$ a $3^{\mathrm{a}}$ série do Ensino Médio e possuindo respectivamente, 80, 72 e 66 
estudantes. Além disso, 37 estudantes foram classificados como "sem série" pois não realizam todas as disciplinas de uma mesma série, seja por motivos de aproveitamento, reprovação ou até mesmo transferência.

A partir das notas dos estudantes foi calculada a mediana de ambos os gêneros por área de conhecimento. A mediana do Núcleo Profissional Informática resultou em 8 para o gênero feminino e 7,9 para o masculino. Já nas áreas de Matemática e Ciências da Natureza foi 7,3 para as meninas e 7 para os meninos. Na área de Ciências Humanas as medianas foram 8,5 no gênero feminino e 8 no gênero masculino, em Linguagens e Códigos o resultado foi 8,3 para estudantes do gênero feminino e 7,7 para estudantes do gênero masculino, e por último na matérias que compõem o núcleo articulador a mediana foi de 7,9 e 7,8 para meninas e meninos, respectivamente. Tais resultados auxiliam na desconstrução do estereótipo de que mulheres não são boas em ciências exatas e em computação, visto que, se homens e mulheres são igualmente capazes e possuem rendimentos estatisticamente iguais no Núcleo Profissional Informática, espera-se que a adesão a uma graduação em computação possa ser semelhante para ambos os gêneros. Porém, essa adesão não vem sendo verificada no cenário nacional, tendo em vista os estudos apresentados anteriormente.

Por meio das notas, foi calculada a média aritmética para cada uma das áreas do conhecimento por gênero e inserido o desvio padrão para indicar o quanto as notas dos estudantes variam entre si. O gráfico das médias das áreas de conhecimento, sendo: Ciências da Natureza (C.N.); Ciências Humanas (C.H.); Linguagens e Códigos (Ling.); Matemática (Mat); Núcleo articulador (Articulador) e Núcleo Profissional (Profissional) e seu respectivo desvio padrão agrupado por gênero (Figura 2) mostra que, mesmo sendo menor em números totais, as estudantes do gênero feminino possuem média superior em todas as áreas de conhecimento e uma menor variação entre as notas, comparado ao estudantes do gênero masculino.

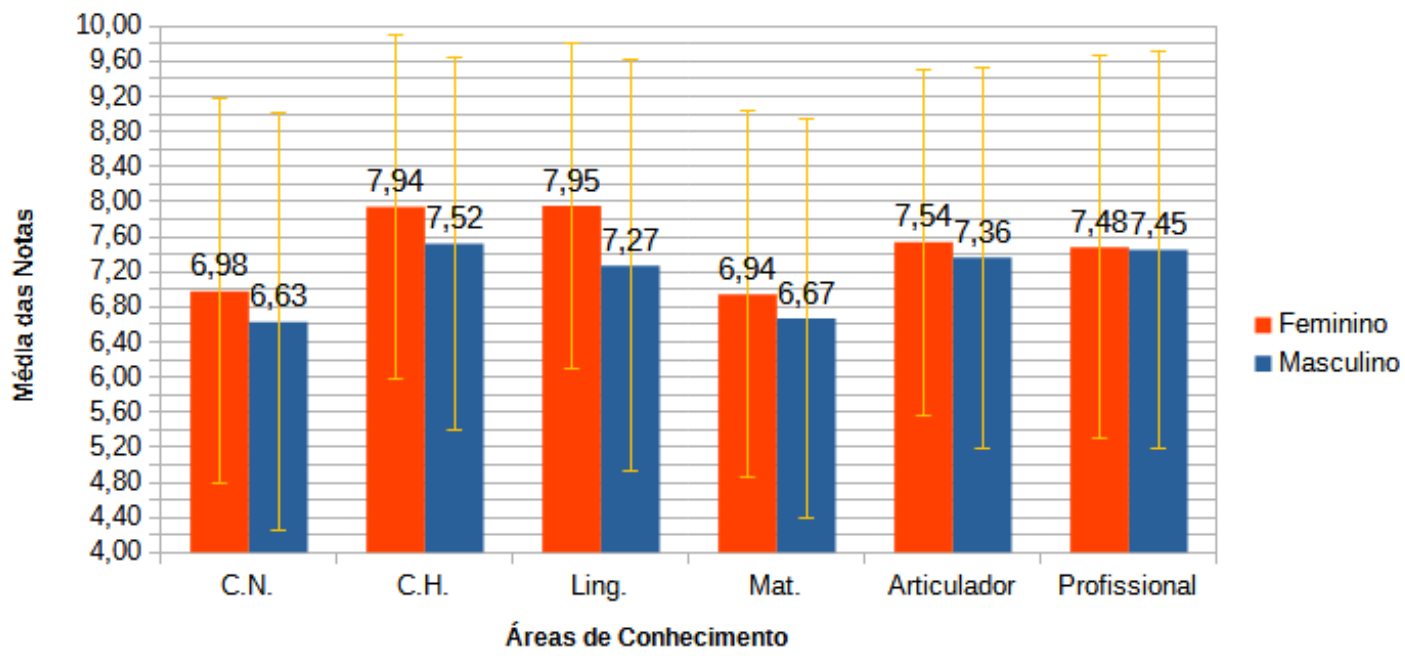

Figura 2. Gráfico das médias das áreas de conhecimento e desvio padrão de cada média dividido por gênero. Fonte: Própria. 
Para aferir de forma estatística a diferença entre os grupos, adotou-se o uso de testes de hipótese. Os testes realizados foram o Teste F, que faz a comparação das variâncias de duas amostras a fim de indicar se há diferença entre elas, e o Teste $\mathrm{T}$ de Student, que realiza a comparação das médias de duas amostras, tendo em vista que existem dois possíveis Teste $\mathrm{T}$ para duas amostras independentes, fez-se necessário a aplicação prévia do Teste $\mathrm{F}$ para aferir se as amostras são supostamente iguais ou diferentes e então efetuar o Teste T correto.

Com as notas de cada área de conhecimento dos gêneros feminino e masculino, os testes $\mathrm{F}$ e $\mathrm{T}$ foram adotados com o objetivo de verificar se existe ou não diferença estatisticamente significativa entre as notas de meninas e meninos em cada área de conhecimento e os resultados obtidos encontram-se na Tabela 1. Como é possível observar nas áreas de conhecimento de Ciências da Natureza, Matemática, Ciências Humanas e Linguagens e Códigos há uma diferença estatisticamente significativa entre o desempenho de meninos e meninas evidenciado pelo valor resultante dos testes aplicados.

É possível afirmar tal diferença por meio do valor-p do teste de variâncias (Teste F) nas áreas de Ciências da Natureza, Matemática, Ciências Humanas, Linguagens e Códigos e Núcleo Articulador, que sendo menor que 0,05, nível de significância, indica que as amostras são supostamente diferentes e com a aplicação do Teste $T$ e o valor-p sendo também menor que o nível de significância estabelecido confirmou essa diferença nas áreas de conhecimento citadas acima, exceto no Núcleo Articulador, pois com a aplicação do Teste $\mathrm{T}$ e o resultado obtido maior que 0,05 indica que as amostras são equivalentes, de forma que a hipótese nula de que não existe diferença entre as notas é aceita.

Tabela 1. Valor-p do teste $\mathrm{F}$ e teste $\mathrm{T}$ em cada área de conhecimento.

\begin{tabular}{|l|c|c|}
\hline \multicolumn{1}{|c|}{ Área de conhecimento } & Teste F & Teste T \\
\hline Ciências da Natureza & 0,0012891 & 0,0000055640704 \\
\hline Matemática & 0,04346307 & 0,021314809565 \\
\hline Ciências Humanas & 0,00001614 & 0,000000000000042 \\
\hline Linguagens e Códigos & 0,0000000000000114 & 0,00000000000000032 \\
\hline Núcleo Articulador & 0,03453014 & 0,09684814066 \\
\hline Núcleo Informática & 0,180070532 & 0,35933054670 \\
\hline
\end{tabular}

Considerando a questão de pesquisa levantada, a partir dos resultados obtidos é possível afirmar que existe diferença no rendimento acadêmico entre os gêneros masculino e feminino no curso de nível médio técnico analisado no presente estudo, uma vez que as estudantes do gênero feminino obtiveram média superior em todas as seis áreas de conhecimento observadas e com a aplicação dos teste estatísticos é 
possível afirmar que essa diferença é estatisticamente significativa nas seguintes áreas de conhecimento: Matemática, Ciências Humanas, e Linguagens e Códigos. Tais resultados dialogam com os dados obtidos nos trabalhos de Marques et al. (2021) e de Pereira et al. (2020), visto que ambos estudos obtiveram resultados semelhantes ao observado na presente pesquisa, uma vez que a análise do rendimento acadêmico e das médias disciplinas apontam que estudantes do gênero feminino possuem desempenho levemente superior ao gênero masculino.

\section{Considerações finais}

Este artigo apresenta dados sobre o rendimento escolar por gênero em seis áreas de conhecimento de todos estudantes regularmente matriculados em um Curso Técnico em Informática para Internet Integrado ao Ensino Médio. Tendo em vista que a escolha feminina por uma graduação em Computação vai muito além de uma simples afinidade pela área, este estudo corrobora no sentido de influenciar positivamente para que meninas se sintam mais confiantes e optem por seguir carreira em TI, ao mesmo tempo que demonstra que o rendimento acadêmico não é o principal fator determinante para evasão na área.

Foi verificado que estudantes do gênero feminino possuem média aritmética levemente superior ao gênero masculino em todas as áreas de conhecimento, porém com a aplicação do Teste F e Teste T de Student é possível concluir que tal diferença é estatisticamente significativa nas áreas de conhecimento de Ciências da Natureza, Matemática, Ciências Humanas, e Linguagens e Códigos. Tais resultados respondem à questão de pesquisa norteadora deste estudo de que há diferença entre o rendimento de meninas e meninos, visto que as estudantes do gênero feminino possuem média superior nas seis áreas de conhecimento analisadas. Provando a importância de atividades que incentivem a participação feminina em TI ainda no ensino médio, e de suporte às estudantes a fim de que sigam carreira na área por meio de um curso de graduação em computação.

Outrossim, os resultados apresentados nesta pesquisa auxiliam na desconstrução do estereótipo de que mulheres possuem desempenho inferior quando comparadas aos homens, principalmente quanto a ciências exatas e consequentemente em computação. E assim servem como subsídios para discussões, visto que, se homens e mulheres são igualmente capazes e possuem rendimentos estatisticamente iguais, em especial, na área de conhecimento do Núcleo Profissional Informática, espera-se que o ingresso em uma graduação em computação seja semelhante para ambos os gêneros. Porém, essa adesão não vem sendo verificada no cenário nacional, tendo em vista dados da Sociedade Brasileira de Computação (SBC) demonstrando que apenas 13,92\% dos concluintes de cursos de graduação em computação em 2019 são do gênero feminino e somente 14,8\% dos ingressantes no mesmo ano são mulheres (NUNES, 2019).

Para trabalhos futuros, objetiva-se realizar uma análise qualitativa por meio de entrevista estruturada com as estudantes egressas do respectivo curso de nível médio no sentido de se averiguar os motivos que as influenciaram para que escolhessem ou não a 
carreira de computação, a fim de traçar um perfil cultural e socioeconômico das estudantes. Além disso, podem ser realizadas análises do rendimento acadêmico em cada um dos anos do ensino médio, com o objetivo de verificar se existem diferenças no desempenho acadêmico em cada ano do curso. Estas análises dialogam com o relatório do PISA, pois mesmo que as meninas tenham maior rendimento acadêmico em todos os níveis de conhecimento, é necessário autoconfiança para permanecer na área de computação.

\section{Agradecimentos}

Agradecemos ao IF Goiano - Campus Ceres por todo o apoio para o desenvolvimento da presente pesquisa que foi fruto do Programa de Institucional de Bolsas de Iniciação Científica (PIBIC), ofertado pela instituição e desenvolvido durante o ciclo 2020/2021. E também ao Núcleo de Estudos e Pesquisa em Tecnologia da Informação (NEPeTI) e ao projeto Meninas Digitais no Cerrado pelo suporte técnico, acadêmico e psicológico durante todo o decorrer da pesquisa e produção deste artigo.

\section{Referências}

INEP. Censo da Educação Superior. Notas estatísticas. Ministério da Educação Instituto Nacional de Estudos e Pesquisas Educacionais Anísio Teixeira, 2017.

LOUZADA, N.; SANTANA, T.; ASSIS, I.; BRAGA, R.; BRAGA, A. Agindo sobre a diferença: atividades de empoderamento feminino em prol da permanência de mulheres em cursos de Tecnologia da Informação. In: WOMEN IN INFORMATION TECHNOLOGY (WIT), 13., 2019, Belém. Anais do XIII Women in Information Technology. Porto Alegre: Sociedade Brasileira de Computação, july 2019. p. 69-78.

MARQUES, D.; SILVA, A. C. da; GUEDES, G. B.; SANTOS JUNIOR, C. R. dos. Desempenho Acadêmico e o Ingresso no Curso Superior: uma análise das estudantes ingressantes entre 2016 a 2020 do curso Técnico em Informática Integrado ao Ensino Médio. In: WOMEN IN INFORMATION TECHNOLOGY (WIT), 15., 2021, Evento Online. Anais [...]. Porto Alegre: Sociedade Brasileira de Computação, 2021. p. 51-60. ISSN 2763-8626. DOI: https://doi.org/10.5753/wit.2021.15841.

MENINAS DIGITAIS (2021). Meninas Digitais. Disponível em: $<$ https://meninas.sbc.org.br/programa-meninas-digitais-apresenta-relatorio-tecnico-d o-periodo-2019-2020-durante-o-csbc-2020/>. Acesso em: 10 de setembro de 2021.

NUNES, D.J. Educação Superior em Computação, Estatísticas 2019. Sociedade Brasileira de Computação-SBC. Disponível em: <https://www.sbc.org.br/ documentos-da-sbc/send/133-estatisticas/1354-educacao-superior-em-computacao-es tatisticas-2019>. Acesso em: 20 de julho de 2021. 
PEREIRA, J. S.; DE MOURA, L. R.; COELHO, N. M. A.; MONTES, G. C.; MAGESTI, I. O.; COELHO, A. M.. Uma Análise da Participação das Mulheres nos Cursos Técnico em Informática e Ciência da Computação do Instituto Federal do Sudeste de Minas Gerais. In: WOMEN IN INFORMATION TECHNOLOGY (WIT), 14. , 2020, Cuiabá. Anais [...]. Porto Alegre: Sociedade Brasileira de Computação, 2020. p. 139-148. ISSN 2763-8626.DOI: https://doi.org/10.5753/wit.2020.11284.

PISA EM FOCO. O que está por trás da desigualdade de gênero na educação?. Disponível em: <https://www.oecd.org/pisa/pisaproducts/pisainfocus/PIF-49\%20 (por).pdf>. Acesso em: 24 mai. 2020.

RESENDE, D. G.; GOMES JR, L. C.; BERARDI, R. C. G.. A influência da diversidade de gênero e da sociabilidade no desempenho acadêmico de equipes de estudantes de graduação em Computação - uma análise preliminar. In: SIMPÓSIO BRASILEIRO DE INFORMÁTICA NA EDUCAÇÃO, 31. , 2020, Online. Anais [...]. Porto Alegre: Sociedade Brasileira de Computação, 2020. p. 1363-1372. DOI: https://doi.org/10.5753/cbie.sbie.2020.1363.

RIBEIRO, K. da S. F. M.; MACIEL, C.. Fatores de Influência na Escolha pela Continuidade da Carreira em Computação pelas Estudantes de Ensino Médio Técnico em Informática. In: WOMEN IN INFORMATION TECHNOLOGY (WIT), 14. , 2020, Cuiabá. Anais [...]. Porto Alegre: Sociedade Brasileira de Computação, 2020. p. 40-49. ISSN 2763-8626. DOI: https://doi.org/10.5753/wit.2020.11274.

RIBEIRO, K. S. M.; MACIEL, C.; BIM, S. A. Relatório do Programa Meninas Digitais para o período 2018-2019. Sociedade Brasileira de Computação. Relatório. Porto Alegre, 2019, 12p.

SANTOS, C. M. Por que as mulheres "desapareceram" dos cursos de computação?. 2017. Disponível em: <https://jornal.usp.br/universidade/por-que-as-mulheresdesapareceram-dos-cursos-de-computacao/>. Acesso em: 24 de maio de 2020.

TEIXEIRA, A.; POSSER, C. V. Mulheres que aprendem informática: Um estudo de gênero na área de TI. In: Anais do Workshop de Informática na Escola. 2016. p. 707. 